\section{P108 (continued)}

Background: In 2019, the Society of Nutrition Education and Behavior recommitted to representing the growing demographic of older adults. One metric to assess this commitment may be an equitable representation of articles published in the Society's journal.

Objective: The aim of this retrospective study was to determine the historic representation of the older adult life stage in articles published in the Journal of Nutrition Education and Behavior (JNEB).

Study Design, Setting, Participants: Research, research briefs, and report articles published in JNEB from 1969 to December 2020 were examined to determine the targeted life stage, including older adults ( $>65$ years), adults, young adults (college-age), and infants, children and adolescents. Articles, in which older adults were included as part of a greater population, were also quantified. An "other" category included articles that did not target or state a life stage. Measurable Outcomes/Analysis: The percentage of research articles, research briefs and reports targeting the older adult life stage published in JNEB.

Results: Of the 2,454 articles reviewed, 39\% of research articles, $51 \%$ of research briefs, and $29 \%$ of reports targeted infants, children and adolescents. Articles specifically addressing older adults had the least representation and included only $4 \%$ of research articles, $3 \%$ of research briefs, and $4 \%$ of reports. Older adults as part of a greater population, were represented by $8 \%$ of research articles, $9 \%$ of research briefs, and $5 \%$ of reports. Of note, $37 \%$ of articles fell into the "other" category.

Conclusion: Historically, the older adult life stage is underrepresented in articles published in JNEB. Given the Society's recent resolution to increase the focus on the educational needs of a growing aging population, an increase in journal publications targeting older adults is warranted, and thus equitably represent all life stages in future issues.

Funding: None.

\section{P109 Responsive Feeding During COVID-19: Evaluation of a Specialized Training for ECE Providers and Stakeholders.}

DiptiA.Dev,PhD, ddev2@unl.edu, University of NebraskaLincoln, 255 Louise Pound Hall, 512 N 12 St, Lincoln, NE, 68588; Saima Hasnin, MS, University of Nebraska-Lincoln; Madeleine Sigman-Grant, PhD, RD, University of Nevada Cooperative Extension; Janice Fletcher, PhD, University of Idaho; Jill Cox, RD, MS, Penn State Extension

Background: Practicing family style meal service in early care and education (ECE) settings supports children's autonomy and improves self-regulation of energy intake. However, during COVID-19, CDC passed a directive to ECE to pause family style meal service. Therefore, we conducted an ECE webinar focused on how to feed children responsively during plated meal service to help providers support children's autonomy and self-regulation.

Objective: To evaluate changes in the level of understanding, behavioral intention to implement knowledge, and confidence about how to feed children (2-5 years) responsively during plated meal service. Also, to determine need for future training opportunities.

Study Design, Setting, Participants: Retrospective pretest-posttest study. The webinar was advertised through an email newsletter disseminated by a nationallevel platform, Penn State Extension Better Kid Care. Participants attended the online webinar for 1 hour on August 11, 2020, had access to an Extension publication (http://bit.ly/3pnJ71X) and responded to a follow-up survey (Qualtrics Link: http://bit.ly/3jMWvvv). Participants identified themselves as either ECE providers $(n=77)$ or other stakeholders $(n=30)$, who can offer opportunities or guidance to providers.

Measurable Outcome/Analysis: Change in self-reported evaluation score (range $1=$ low to $7=$ high) for understanding, behavioral intention to implement knowledge, and confidence about how to feed children responsively during plated meal service. Paired sample $t$ tests (alpha $=0.05)$ followed by Sidak-Bonferroni correction (adjusted $P=0.007$ ).

Results: Both providers and stakeholders reported significantly higher $(P<0.001)$ level of understanding, intention to implement knowledge obtained, and confidence regarding implementing responsive feeding during plated meal service after the webinar compared to before the webinar. Participants expressed need for online nutrition trainings with emerging themes such as feeding infants, safe food preparation and storage, flexibility of CACFP rules, and physical distance during meals.

Conclusion: Intentional mealtime conversations, giving children small tasks supporting their autonomy, and setting clear expectations while adjusting mealtime routines can offer a promising avenue for implementing responsive feeding during plated meal service.

Funding: None.

\section{P110 The Healthy Communities Initiative: Gathering Community Input During COVID-19}

Jessica Stroope, MPH, jstroope@agcenter.lsu.edu, Louisiana State University AgCenter, 202G Knapp Hall, Baton Rouge, LA, 70803; Matthew Greene, LDN, MSPH, RD, Louisiana State University AgCenter; Katherine Seal, MPH, Louisiana State University AgCenter; Denise Holston, PhD, $L D N, R D N$, Louisiana State University AgCenter

Background: The Louisiana Healthy Communities Initiative, led by the LSU AgCenter, guides SNAP-Ed policy, systems, and environmental change efforts. Prior to COVID19, agents held in-person community forums to gather input and community priorities for interventions. COVID19 spurred innovation in this process. Although many states have used videoconferencing services to host meetings, lack of functional internet access posed a barrier for many communities in Louisiana.

Objective: To gather broad community input through Qualtrics surveys in order to assess needs and assets, guide project prioritization, and gather feedback on previous projects.

Continued on page 576 


\section{P110 (continued)}

Study Design, Settings, Participants: Qualtrics surveys were distributed via email to community members, stakeholders, and partners serving low-income audiences. Three surveys solicited feedback for existing Healthy Communities coalitions $(n=45) ; 1$ survey gathered initial input for a newly formed Healthy Communities coalition $(n=63)$. The overall response rate across all surveys was $23.6 \%$.

Measurable Outcome/Analysis: Survey responses to community health and engagement questions were separated by counties and analyzed through qualitative content analysis. The research team worked with Extension agents to conduct member checking through discussing survey results with participants.

Results: One hundred and eight responses were received from 4 communities. Extension agents reported high satisfaction with the process. Surveys provided action items and potential new coalition members. Across all 4 communities, common themes included acknowledging racial health disparities, a need for greater community involvement in coalition efforts, and a lack of healthy food options.

Conclusion: Qualtrics surveys were an effective way to gather community input and allowed wider participation than would have been possible with a virtual meeting. Post-COVID, agents plan to continue to solicit community input through Qualtrics surveys, in addition to faceto-face forums. This method is a valuable tool for lowerincome and rural communities. Results reflected an awareness of low community engagement, racial health disparities and limited healthy food access.

Funding: Supplemental Nutrition Assistance Program Education.

\section{P111 The Impact of a Culinary Medicine Course on the Nutrition Care Attitudes of Pre-Health Profession Students}

Sarah Albrecht,BA, BS, saraha@udel.edu, University of Delaware, 210 South College Ave, Newark, DE, 19716; Kristin Wiens, MS, LDN, RDN, University of Delaware

Background: Studies suggest cooking skill-based learning increases knowledge and improves nutrition attitudes and confidence more than traditional knowledge-based approaches. Current educational strategies may not adequately prepare future healthcare professionals to become empathetic and innovative problem-solvers who have the skills to provide evidence-based and practical advice about nutrition.

Objective: The objective of this study was to investigate how a culinary medicine course for pre-health profession college students impacts nutrition care attitudes.

Study Design, Setting, Participants: Twenty-five prehealth profession undergraduate students enrolled in an interprofessional, experiential culinary medicine course participated. This was a skills- and case-based course which focused on food and cooking, and evidence-based nutrition recommendations for chronic disease.

Measurable Outcome/Analysis: Items from the validated Nutrition in Patient Care Survey (NIPS) were administered pre-post course completion to assess nutrition care attitudes. Sociodemographic characteristics such as age, year in college, career aspirations, race, and ethnicity were collected. Data were analyzed using descriptive statistics, paired $t$ tests, Wilcoxon signed rank tests, and ANOVA with post hoc analysis.

Results: Baseline attitudes scores were somewhat high compared to literature from working health professionals. No significant changes were seen in attitude scores, but differences were found based on ethnicity and career aspirations. Students identifying as Hispanic had more negative attitudes regarding nutrition counseling $(P=0.001)$ and including nutrition in routine patient care $(P=$ 0.013), but had more positive attitudes towards the importance of taste in improving dietary patterns $(P=0.002)$. Aspiring doctors, dentists, and physician assistants had more negative attitudes about nutrition counseling than future dietitians $(P=0.008)$ and nurses $(P=0.049)$.

Conclusion: Although no significant changes were found in attitude scores, the differences based on ethnicity and career aspirations warrant further investigation. Nutrition care is incidental to many health professionals' practice. Strategies to cultivate positive attitudes regarding the role of nutrition across health disciplines should be identified to better develop competency.

Funding: None.

\section{Physical Activity}

\section{P112 Partnering for Community-Health and Wellness: A County-Wide, Mayor's Fitness Challenge}

Angelika Keene, BS, University of Florida; Elizabeth Shephard, BS, MPA, bshephar@ufl.edu, University of Florida, 3695 Lake Dr, Cocoa, FL, 32926

Objective: To increase physical activity in citizens utilizing a competition between cities and other partnerships.

Use of Theory or Research: Knowing that accountability is a major factor in increasing physical activity, the Mayor's Fitness Challenge is a competition designed to promote health and wellness and provide accountability and positive reinforcement.

Target Audience: Citizens of all ages living in Brevard County.

Program Description: To mitigate organizational challenges, this program takes a county-wide approach and utilizes partnerships to provide opportunities for multiple cities and organizations to address community health and wellness via a challenge that shares resources, funding, personnel, and expertise. Eleven of the 16 municipalities participated in a free, 8-week fitness challenge designed to get citizens involved in a healthy competition. Everyone who joins within a municipality is considered part of that mayor's "team." The only requirement is participants must start moving and track their progress. The committee uses a point system to equalize different team sizes. The municipality with the most points is awarded 'Most

Continued on page $S 77$ 